
\title{
28 Research Suare \\ Identification of key genes associated with Idiopathic Pulmonary Fibrosis by combining multiple analytical methods
}

\section{Yong Zhao}

Tianjin Chest Hospital https://orcid.org/0000-0002-2237-5431

\section{Shengnan Yang}

Tianjin Chest Hospital

\section{Weigang Dong}

Tianjin Chest Hospital

\section{Yuechuan Li}

Tianjian Chest Hospital

Jing Feng ( $\square$ fengjingdoctor@gmail.com )

Tianjin Medical University General Hospital https://orcid.org/0000-0003-0604-3581

\section{Research}

Keywords: Idiopathic Pulmonary Fibrosis, Least absolute shrinkage and selection operator, support vector machine, Receive operator curves analysis

Posted Date: October 28th, 2020

DOl: https://doi.org/10.21203/rs.3.rs-96044/v1

License: (c) (i) This work is licensed under a Creative Commons Attribution 4.0 International License. Read Full License 


\section{Abstract}

Idiopathic Pulmonary Fibrosis (IPF) is the most common type of idiopathic interstitial pneumonias (IIPs) with unclear etiology and lack of effective diagnostic biomarker or therapeutic targets. This study aimed to identify the key genes associated with IPF through multiple microarray datasets. two datasets including GSE32537 and GSE110147 retrieved from Gene Expression Omnibus (GEO) database were used as training dataset $(\mathrm{N}=169)$ and external validation dataset $(\mathrm{N}=33)$. A total of 365 differentially expressed genes (DEGs) were identified between normal tissue and IPF tissue in the training dataset. Function enrichment analysis result indicated that these genes were involved in cell proliferation, response to stimulus, cellular process and immune system process. We then constructed a protein-protein network (PPI) using the cytoscape software based on the DEGs and the hub modules were screened using the MCODE algorithm. Then the DEGs from hub modules were further exploited into Least absolute shrinkage and selection operator (LASSO) and support vector machine (SVM) analysis in the training dataset. 13 key genes including SERPIND1, CDH2, CP, WDR63, DNAH5, FGG, THBS1, VCAM1, COL1A2, POSTN, CXCL12, MMP13, SERPINE1 were further validated in the validation dataset. In addition, we also applied the CIBERSORT algorithm to evaluate the infiltration level of immune cells in IPF tissue, and the correlation between key genes and infiltrating immune cells. We identified that Plasma cells, CD 4 naive T cells, NK resting cells, Monocytes, Mast resting cells and Neutrophils might involved in the process of IPF. Moreover, we also identified several drugs including pregnenolone, indoprofen, ramipril, inburnine may contribute to the treatment of IPF. In conclusion, the 13 genes might serve as potential diagnostic markers of IPF, and the immune cell infiltration plays an essential role in the occurrence and progression of IPF.

\section{Background}

Idiopathic pulmonary fibrosis (IPF) is a chronic and irreversible lung fbrosis, which is characterized by diffuse alveolitis and structural disorder of alveoli [1]. Despite the prevalence of IPF is not high, the incidence has been increased. The cases of the IPF is about 2.8 and 18 per year for per 100,000 people in Europe and North America, respectively [Hutchinson et al.]. The diagnostic age of the IPF is about 65 years and the incidence rate of IPF in the men were higher that women [2]. Recently, although some potential biomarkers associated with IPR have been identified, the prognosis of IPF still limited. Thus, it's in urgently need that understanding the mechanism of the IPF pathogenesis.

Accumulated evidence have been revealed that microarray datasets were used to identify a large number of biomarkers in variety of disease based on the molecular levels $[3,4]$ Wang et al took advantage of six microarray datasets including GSE10667, GSE15197, GSE21369, GSE24206, GSE32537 and GSE110147 to identify 2 key modules (yellow and black) and hub genes that associated with the trait of IPF [5]. Fan et al combined microarray dataset (GSE32538) and mRNA datasets (GSE32537, GSE53845, and GSE10667) to analyzed potential microRNAs and genes associated with idiopathic pulmonary fibrosis (IPF) [6]. Moreover, IPF has been reported that associated with variety of putative miRNAs, such as miR92a, miR-210 $[7,8]$. Although some potential key genes or miRNAs have been identified, considering the complexetiology of the IPF, it is far from enough and more accurately biomarkers are needed. 
The present study is aimed to identify key genes and immune cell infiltration associated with IPF. By performing differentially expression analysis in the training dataset, the significantly genes including upregulated and down regulated genes were screened. The network was then constructed on the basis of DEGs and the key genes were screened and validated by usingLeast absolute shrinkage and selection operator (LASSO) and support vector machine (SVM) analysis in the training dataset and validation dataset. Moreover, the landscape of immune cell infiltration in the IPF was estimated using the CIBERSORT algorithm. At last, we explore the association between key genes and infiltrating immune cells to better understanding the molecular immune mechanism during the development of IPF.

\section{Materials And Methods}

\section{Datasets collection}

The gene expression datasets associated with idiopathic pulmonary fbrosis (IPF) were downloaded from Gene Expression Omnibus(GEO) database (http://www.ncbi.nIm.nih.gov/geo/). Queries were performed using "IPF tissue\& normal tissue" as a keyword. The search was restricted to the following specific region: study type: expression profiling by array, and species: "Homo sapiens". As a result, six datasets including GSE32537 and GSE110147 were fitted in our study $[9,10]$.

\section{Differentially expression analysis, network construction and potential drugs identification}

The differentially expressed genes (DEGs) were screened between IPF tissue and normal tissue by conductinglimma analysis with the cut-off: $|\log \mathrm{FC}|>1$ and adjusted $p$ value $<0.05$ [11]. Moreover, The relationship among the DEGs was identified and visualized using the String database and cytoscape software, respectively [12]. The top 2 hub modules were further screened using the MCODE algorithm. The potential drugs were identified using the CMAP database based on the DEGs [13].

\section{LASSO analysis and SVM analysis}

After the selection of hub modules, a Least Absolute Shrinkage and Selector Operation (LASSO) algorithm, with penalty parameter tuning conducted by 10 -fold cross-validation, was performed to screening potential key genes from hub modules . In addition, another algorithm, Support Vector Machine-Recursive Feature Elimination (SVM-RFE), was also applied to select key genes [14]. Finally, we combined the genes from either LASSO or SVM-RFE algorithms for further analysis. A two-sided $p<0.05$ was considered to be statistically significant.

\section{Estimation of immune cell infiltration}

The gene expression profile data was uploaded to the CIBERSORTx online tools [15]. The LM22 signature was selected as a reference file which defended 22 immune cell, including B cells, plasma cells, T cells, mast cells, natural killer (NK) cells, eosinophils, neutrophils and monocytes. Using bulk-mode batch correction, the absolute proportion of each cell type in the mixture was obtained, and only the samples with $p$ value smaller than 0.05 was exploited to the downstream analysis. 


\section{Statistically analysis}

All statistical tests were implemented by R/3.5.2 using a Wilcoxon test (Mann-Whitney test) for continuous data. For all statistical analysis, a $\mathrm{P}$ value smaller than .05 was considered as statistically significant.

\section{Result}

\section{Differentially expression analysis between normal tissue and IPF tissue}

In the present study, the gene expression profile dataset GSE32537 and GSE110147 were employed as the training dataset and external dataset. To identified differentially expressed genes between the IPF tissue and normal tissue, we conducting a limma analysis with a screening criterion: $|\log 2 \mathrm{FC}|>1$ and Adjusted $p$ value $<0.05$. As a result, a total of 365 DEGs were identified, including 119 down-regulated genes and 246 up-regulated genes (Figure 1A-B). Among these genes, we also discovered some of genes that have been found to be associated with IPF were presented in our result, such as KRT5, BPIFB1 andAGER.The GO enrichment analysis result revealed that the genes involve in response to growth factor, blood vessel development, cell-substrate adhesion and epithelial cilium movement and cell adhesion molecule binding. The pathway enrichment analysis showed that genes in IPF mainly involved in ECMreceptor interaction, Cytokine-cytokine receptor interaction and Protein digestion and absorption.

\section{Protein-Protein network construction}

To constructed a protein-protein network, we uploaded all DEGs to the string database and set median confidence to 0.400 . We then obtained 1037 edges and 308 nodes (Figure 2A). The hub modules was identified by using the MCODE algorithm with the degree more than 2. AS a result, we selected the top two modules with 37 genes including DNAH3, HYDIN, RSPH1, DRC1, SERPIND1, MATN3, TNC, CDH2, GOLM1, LTBP1, DNAH11, CP, DNAH10, DNAH9, DNAH12, DNAH6, DNAAF1, RSPH4A, DNAH7, WDR78, ARMC4, WDR63, DNAH5, DNAI1, FGA, FGG, THBS1, COL1A1, VCAM1, COL1A2, POSTN, COMP, CXCL12, MMP13, CTSK, SERPINE1, COL3A1 (Figure 2B).

\section{Selection of the key genes}

In order to narrow down the selection of the genes from the two hub modules and obtained more reliable key genes, we first performed LASSO analysis to identify 13 genes for IPF (Figure 3A-B). The SVM-RFE algorithm were also applied to identified 29 genes for IPF (Figure 3C). The potential gene markers characterized by the two algorithms overlapped, and finally 13 key genes were selected including SERPIND1, CDH2, CP, WDR63, DNAH5, FGG, THBS1, VCAM1, COL1A2, POSTN, CXCL12, MMP13, SERPINE1 (Figure 3D). The expression of these genes were further identified by another external dataset GSE110147 dataset. As shown in Figure 4, we observed that most of key genes were presented a significant divergence, including SERPIND1, CDH2, CP, WDR63, DNAH5, VCAM1, COL1A2, POSTN, MMP13, suggesting that the robusticity and reliability of our genes. 


\section{Immune Cell Infiltration analysis}

The 22 types of immune cell infiltration level was calculated using the CIBERSORTx algorithm and the sample were retained with the $p$ value $<.05$. The correlation heatmap of 22 immune cells revealed that activated NK cells was most positively correlated with Plasma cells, while resting NK cells was most negatively correlated with activated NK cells (Figure 5A). The violin plot of the immune cell infiltration divergence showed that Plasma cells, CD4 memory resting T cells, regulatory T cells(Tregs), resting Mast cells were significantly high expressed in the IPF tissue, while CD4 naive T cells, Monocytes, Neutrophils were significnatly high expressed in the normal tissue (Figure 5B). Moreover, we also evaluate the relationship between key genes and immune cells. Figure 6 showed that resting Mast cells, Monocytes and Neutrophils were most correlated with all key genes, suggesting that these key genes may regulate the function of resting Mast cells, Monocytes and Neutrophils.

\section{CMAP analysis}

To identify the potential molecular drugs of IPF, the DEGs were uploaded to the Connectivity Map (CMAP) database and matched them with small molecule therapy. The Table 1 listed the potential enriched molecular drugs and corresponding enrichment values with the enrichment value that $<-0.6$. The molecular drugs including clorsulon, thiamine, $\mathrm{AH}-6809$ and altizide were the most enriched in the analysis. Considering the negative enrich potential small molecule drugs could reverse the gene expression induced by IPF, we further exploited the molecular drugs with enrichment value that $<-0.6$ to the downstream analysis. The mechanism of action for the potential drugs were analyzed and mainly enriched in ACE inhibitor, rilmenidine, Adrenergic receptor agonist, Adrenergic receptor antagonist, Antiarrhythmic, Cyclooxygenase inhibitor, Dopamine receptor antagonist, GABA receptor antagonist, Glutamate receptor modulator and Thiazide diuretic (Figure 7).

\section{Discussion}

Idiopathic pulmonary fibrosis (IPF), a progressive, devastating and chronic disease that characterized by fibroblast accumulation, collagen synthesis and glycoprotein [16]. In the present study, we integrated multiple analysis of gene expression to explore the molecular pathogenesis and immune cells infiltration level of IPF. In this study, the differentially expressed genes were screened between IPF and normal tissue using the limma analysis. The pathway enrichment analysis revealed that genes mainly involved in ECMreceptor interaction, Cytokine-cytokine receptor interaction and Protein digestion and absorption pathway, indicating that genes may play an important role in the development of IPF. The cytoscape software was further used to construct a protein-protein network and the MCODE algorithm was applied to identified hub modules. In order to further obtained crucial genes that associated with IPF, we further performed a LASSO and SVM analysis. As a result, 13 genes including SERPIND1, CDH2, CP, WDR63, DNAH5, FGG, THBS1, VCAM1, COL1A2, POSTN, CXCL12, MMP13, SERPINE1 were selected through integrating the LASSO analysis and SVM-RFE analysis result. The LASSO (Least Absolute Shrinkage and Selection Operator) methods is an popular and important regularization in many regression analysis methods (e.g., 
COX regression, logistic regression) [17]. Compared with the linear model, LASSO model can reduce the variable numbers and effectively avoid overfitting. The SVM-RFE is an algorithm of machine learning and the optimal subset was selected via a k-fold cross-validation approach [18]. We further evaluated the expression of the 13 key genes by using an another dataset. Most of the genes have a significant divergence betwwen normal and tumor tissue.

Among the 13 key genes, VCAM1, CXCR12 and MMP13 were reported that directly associated with IPF. Agassandian $\mathrm{M}$ et al has been discovered that the VCAM1 presented a high expression level in the IPF tissue and can serve as a responsive mediator of TGF- $\beta 1$ that partakes in fibroblast proliferation in subjects with IPF [19]. Increasing experimental evidence has been demonstrated that CXCR4/CXCL12 is associated with the pathogenesis of lung fibrosis, and the CXCL12 and its receptor CXCR4 showed a high expression level in the human lung fibroblast [20]. Moreover, the high expression of MMP13 have more likelihood to limit the overall extent of ECM deposition in lung fibrosis [21]. Despite Other genes including SERPIND1, CDH2, CP, WDR63, DNAH5, FGG, THBS1, COL1A2, POSTN, SERPINE1 have not been reported in the IPF, it can act as a important biomarker for other disease. For example, the SERPIND1 was reported that a potential biomarker for the survival prediction of lung squamous cell carcinoma [22]. The low expression of $\mathrm{CDH} 2$ can improved glioma patients overall survival time and may benefit from the temozolomide therapy [23]. In addition, Considering the role of the immunity and immune response in the idiopathic pulmonary fibrosis (IPF) was not unclear presently, we further evaluate the immune cell infiltration level of the IPF tissue. We discovered that the level of Plasma cells, CD4 memory resting T cells, regulatory T cells(Tregs), resting Mast cells were significantly high expressed in the IPF tissue, indicating that these immune cells may induce the development of IPF tissue. Studies have been demonstrated thatregulatory T cells(Tregs) exhibited bifunctional potential, with the ability to spontaneously cause fibroblast proliferation and fibrosis following transfer into unchallenged lungs [24]. Moreover, the pulmonary fibrosis is characterized by an inflammatory response that includes macrophages, neutrophils, lymphocytes, and mast cells, thus, it is no surprised that the level of resting mast cells presented a high expression level in IPF tissue compared to the normal tissue. In addition, we also identified several molecular drugs including pregnenolone, indoprofen, ramipril and inburnine which may contribute to the treatment of IPF tissue. According to the research reports, the ACE inhibitor treatment were associated with slower IPF disease progression [25]. Interestingly, the mechanism of the ramipril was ACE inhibitor, indicated a potential drugs for the treatment of IPF. Despite we performed comprehensive bioinformatics analyses using the gene expression data from two microarray datasets of IPF and confirmed the key genes accuracy by performing ROC analysis, several limitations remain. First, the sample size remain needed to extend. Second, the study lacks validation experiments to validate the expression of key genes. Thus, further experiments should be prepared and conducted to support our findings.

\section{Conclusion}

In conclusion, we identified 13 key genes associated with IPF via integrating multiple analysis methods. We also evaluating the relationship between immune cell infiltration and IPF. We discovered that ramipril 
drug may contribute to the treatment of IPF. These result may contribute to better understanding the molecular mechanism of IPF and provided potential drugs for the treatment of IPF.

\section{Declarations}

\section{Data Availability}

The dataset performed in this study are available from the corresponding author on reasonable requests.

\section{Conflicts of Interest Statement}

The authors declare that there are no conflicts of interest regarding the publication of this paper.

\section{Funding Statement}

None

\section{Author contributions}

Jing Feng designed the study, Shengnan Yang, Yuechuan Li collected the dataset of IPF and revised the manuscript. Yong Zhao and Jing Feng analysis data and wrote the manuscript.

\section{References}

1. Selman M, Thannickal VJ, Pardo A, Zisman DA, Martinez FJ, Lynch JP. Idiopathic Pulmonary Fibrosis Seminars in Respiratory Critical Care Medicine. 2004;64:405-30.

2. Christenson Rd ML. 2012. An Official ATS/ERS/JRS/ALAT Statement: Idiopathic Pulmonary Fibrosis: Evidence-based Guidelines for Diagnosis and Management. Yearbook of Diagnostic Radiology.

3. Cao WJ, Wu HL, He BS, Zhang YS, Zhang ZY. Analysis of long non-coding RNA expression profiles in gastric cancer. World Journal of Gastroenterology:133-139.

4. Saei AA, Omidi Y. A Glance at DNA Microarray Technology and Applications. Bioimpacts Bi. 2011;1:75-86.

5. Wang M, Yao R, Wang Y. 2019a. Silencing of IL13RA2 promotes partial epithelial-mesenchymal transition in hepatocellular carcinoma via ERK signaling pathway activation. FEBS Open Bio.

6. Fan L, Xiaoting Y, Ziling H, Shaoqiang Z, Yongxin Z, Hanjing L, Yu Z, Jin-Fu X, Xuyou Z, Xianghua Y. Analysis of Microarray-Identified Genes and MicroRNAs Associated with Idiopathic Pulmonary Fibrosis. Mediators of Inflammation 2017:1-9.

7. Berschneider B, Ellwanger DC, Baarsma HA, Thiel C, Shimbori C, White ES, Kolb M, Neth P, K?nigshoff M. miR-92a regulates TGF- $\beta 1$-induced WISP1 expression in pulmonary fibrosis. International Journal of Biochemistry \& Cell Biology 53:432-441.

8. Bodempudi V, Hergert P, Smith K, Xia H, Herrera J, Peterson M, Khalil W, Kahm J, Bitterman PB, Henke CAJAJPLCMP. 2014. miR-210 Promotes IPF Fibroblast Proliferation in Response to Hypoxia. 
307:283-94.

9. Yang IV, Coldren CD, Leach SM, Seibold MA, et al. Expression of cilium-associated genes defines novel molecular subtypes of idiopathic pulmonary fibrosis. Thorax. 2013 Dec;68(12):1114-21.

10. Cecchini MJ, Hosein K, Howlett CJ, Joseph M, et al. Comprehensive gene expression profiling identifies distinct and overlapping transcriptional profiles in non-specific interstitial pneumonia and idiopathic pulmonary fibrosis. Respir Res 2018 Aug 15;19(1):153.

11. Ritchie ME, Phipson B, Wu D, Hu Y, Law CW, Shi W, Smyth GK. limma powers differential expression analyses for RNA-sequencing and microarray studies. Nucleic Acids Res. 2015;43(7):e47.

12. Szklarczyk D, Gable AL, Lyon D, Junge A, Wyder S, Huerta-Cepas J, Simonovic M, Doncheva NT, Morris JH, Bork P, Jensen LJ, von Mering C. STRING v11: protein-protein association networks with increased coverage, supporting functional discovery in genome-wide experimental datasets. Nucleic Acids Res. 2019 Jan;47:D607-13.

13. Aliyu M, Soltan GL, Shu-Dong Z, et al. A review of connectivity map and computational approaches in pharmacogenomics[J]. Briefings in Bioinformatics(5):903.

14. Huang M-L, Hung Y-H, Lee WM, Li RK, Jiang B-R. SVM-RFE based feature selection and Taguchi parameters optimization for multiclass SVM classifier. TheScientificWorldJournal. 2014;2014:795624-4.

15. Newman AM, Steen CB, Liu CL, et al. Determining cell type abundance and expression from bulk tissues with digital cytometry[J]. Nature biotechnology. 2019;37(7):773.

16. Ley B, Collard HR, King TEJAJRCCM. 2011. Clinical course and prediction of survival in idiopathic pulmonary fibrosis. 183:431-440.

17. Wu J, Jin S, Gu W, Wan F, Zhang H, Shi G, Qu Y, Ye D. Construction and Validation of a 9-Gene Signature for Predicting Prognosis in Stage III Clear Cell Renal Cell Carcinoma. Frontiers in oncology. 2019;9:152-2.

18. Lin X, Li C, Zhang Y, Su B, Fan M, Wei H. Selecting Feature Subsets Based on SVM-RFE and the Overlapping Ratio with Applications in Bioinformatics. Molecules. 2017;23:52.

19. Marianna A, John, et al. VCAM-1 is a TGF- $\beta 1$ inducible gene upregulated in idiopathic pulmonary fibrosis[J]. Cellular Signalling, 2015.

20. Cui HX, Feng YZ, Gu ZL, et al. Research on CXCL12/CXCR4 biological axis and idiopathic pulmonary fibrosis[J]. Chinese Pharmacological Bulletin. 2010;26(3):298-301.

21. Cabrera $S$, Maciel M, Hernández-Barrientos $D$, et al. Delayed resolution of bleomycin-induced pulmonary fibrosis in absence of MMP13 (collagenase 3). Am J Physiol Lung Cell Mol Physiol. 2019;316(5):L961-76. doi:10.1152/ajplung.00455.2017.

22. Ma H, Tong L, Zhang Q, Chang W, Li F. Identification of 5 Gene Signatures in Survival Prediction for Patients with Lung Squamous Cell Carcinoma Based on Integrated Multiomics Data Analysis. Biomed Res Int. 2020;2020:6427483. Published 2020 Jun 8. 
23. Chen Q, Cai J, Jiang C. CDH2 expression is of prognostic significance in glioma and predicts the efficacy of temozolomide therapy in patients with glioblastoma. Oncol Lett. 2018;15(5):7415-22.

24. Ravi R, Noonan KA, Pham V, et al. Bifunctional immune checkpoint-targeted antibody-ligand traps that simultaneously disable TGF $\beta$ enhance the efficacy of cancer immunotherapy[J]. Nat Commun. 2018;9(1):741.

25. Michael, Kreuter D, et al. Association of Angiotensin Modulators With the Course of Idiopathic Pulmonary Fibrosis.[J]. Chest, 2019.

\section{Tables}

\section{Table 1}

The potential drugs of the IPF that validated using the CMAP database 


\begin{tabular}{|c|c|c|c|c|c|c|c|}
\hline rank & cmap name & mean & $\mathrm{n}$ & enrichment & $\mathrm{p}$ & specificity & $\begin{array}{l}\text { percent non- } \\
\text { null }\end{array}$ \\
\hline 1 & 5279552 & -0.706 & 2 & -0.987 & 0.00042 & 0 & 100 \\
\hline 2 & clorsulon & -0.28 & 4 & -0.881 & 0.00048 & 0.0071 & 50 \\
\hline 3 & АН-6809 & 0.768 & 2 & 0.979 & 0.00074 & 0 & 100 \\
\hline 4 & altizide & 0.504 & 4 & 0.843 & 0.00097 & 0 & 75 \\
\hline 5 & Prestwick-689 & 0.337 & 4 & 0.799 & 0.00306 & 0 & 50 \\
\hline 6 & rilmenidine & -0.314 & 4 & -0.787 & 0.00416 & 0.0069 & 50 \\
\hline 7 & Prestwick-857 & -0.291 & 4 & -0.78 & 0.00483 & 0 & 50 \\
\hline 8 & thiamine & -0.45 & 3 & -0.847 & 0.00709 & 0.0053 & 66 \\
\hline 9 & indoprofen & -0.418 & 4 & -0.748 & 0.00812 & 0.0333 & 75 \\
\hline 10 & harmol & 0.594 & 4 & 0.74 & 0.00871 & 0.0233 & 75 \\
\hline 11 & securinine & -0.521 & 4 & -0.727 & 0.01142 & 0.0633 & 75 \\
\hline 12 & dimenhydrinate & -0.034 & 4 & -0.671 & 0.02604 & 0.0288 & 50 \\
\hline 13 & azaperone & 0.345 & 4 & 0.67 & 0.02612 & 0 & 50 \\
\hline 14 & $\begin{array}{l}\mathrm{N} \text {-acetylmuramic } \\
\text { acid }\end{array}$ & -0.306 & 4 & -0.666 & 0.02829 & 0.0274 & 50 \\
\hline 15 & proxyphylline & 0.331 & 4 & 0.653 & 0.03408 & 0.0182 & 50 \\
\hline 16 & vinburnine & -0.342 & 4 & -0.648 & 0.03648 & 0.078 & 50 \\
\hline 17 & pregnenolone & -0.251 & 4 & -0.639 & 0.04076 & 0.0637 & 50 \\
\hline 18 & ramipril & 0.336 & 4 & 0.635 & 0.04345 & 0.0301 & 50 \\
\hline 19 & hydroquinine & -0.332 & 4 & -0.628 & 0.0474 & 0.0567 & 50 \\
\hline
\end{tabular}

Figures 
A

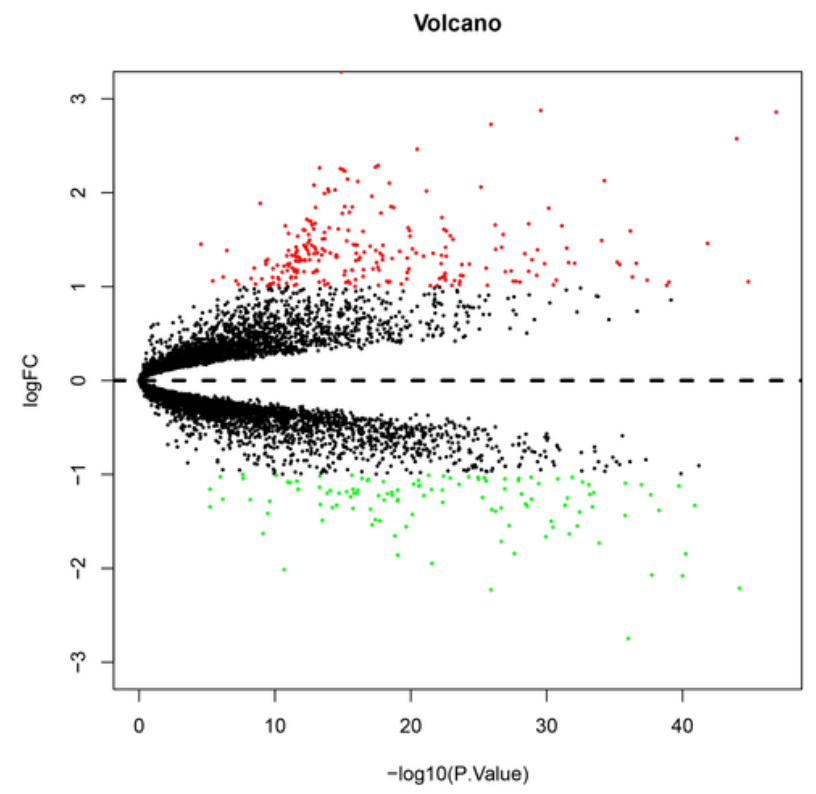

B

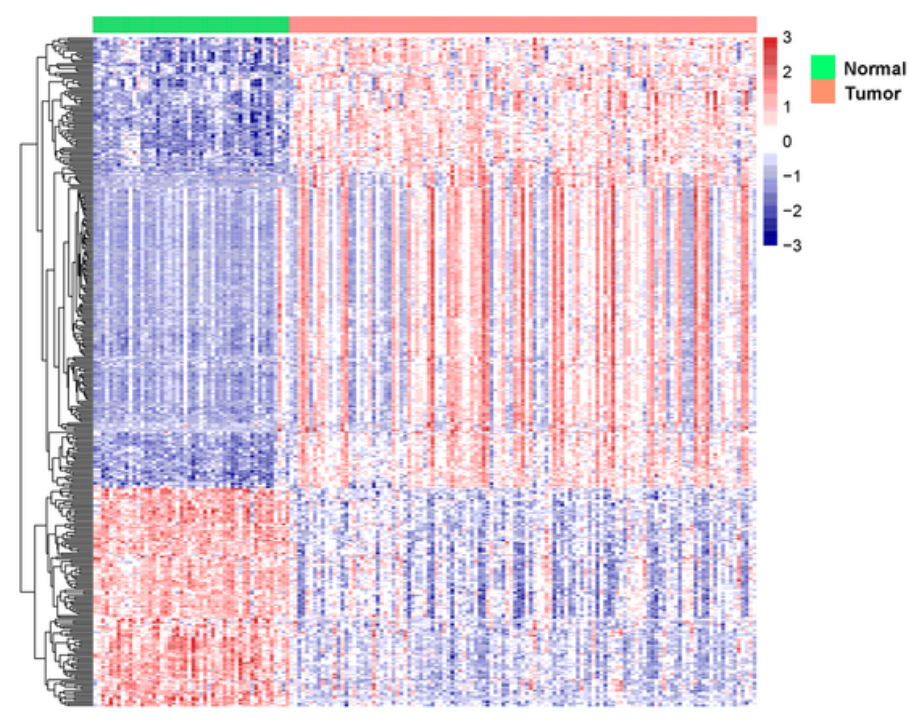

\section{Figure 1}

Differentially expressed analysis between IPF tissue and normal tissue. (A) Volcano plot for the differentially expressed genes, the red dot represent up-regulated genes and green dots represented downregulated genes. (B) A heatmap of the differentially expressed genes.

A

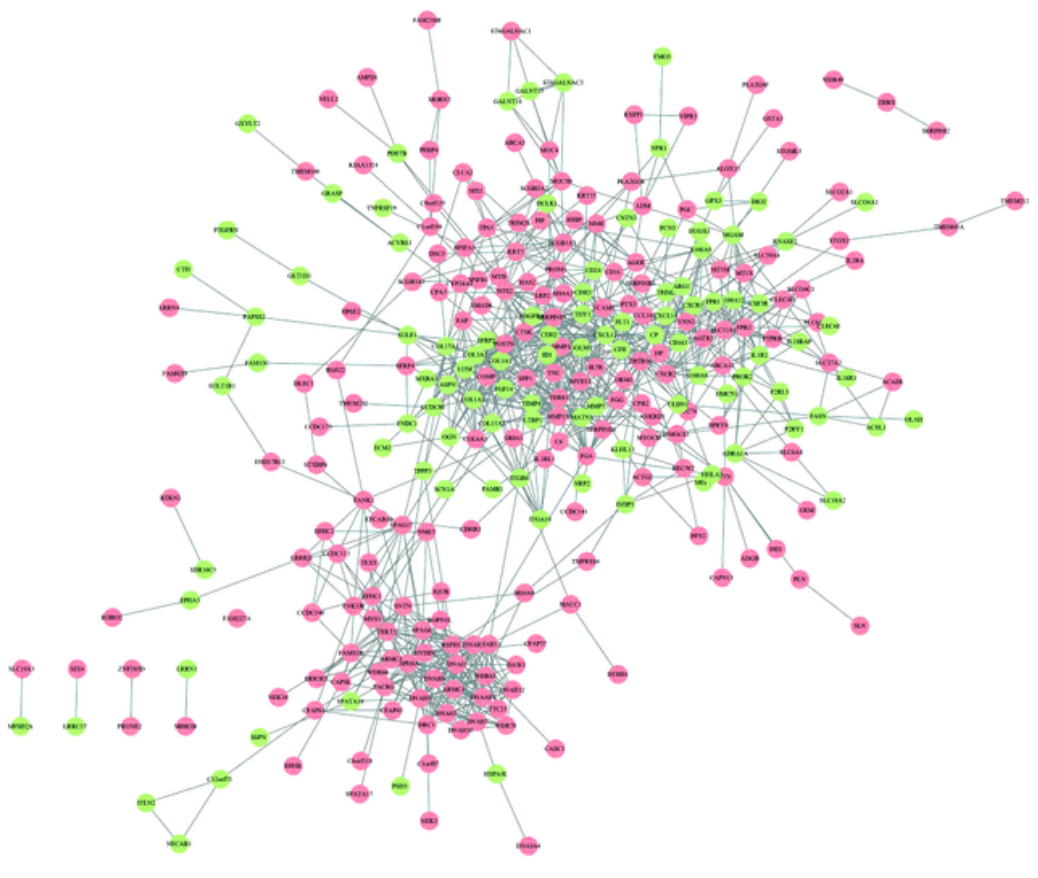

B

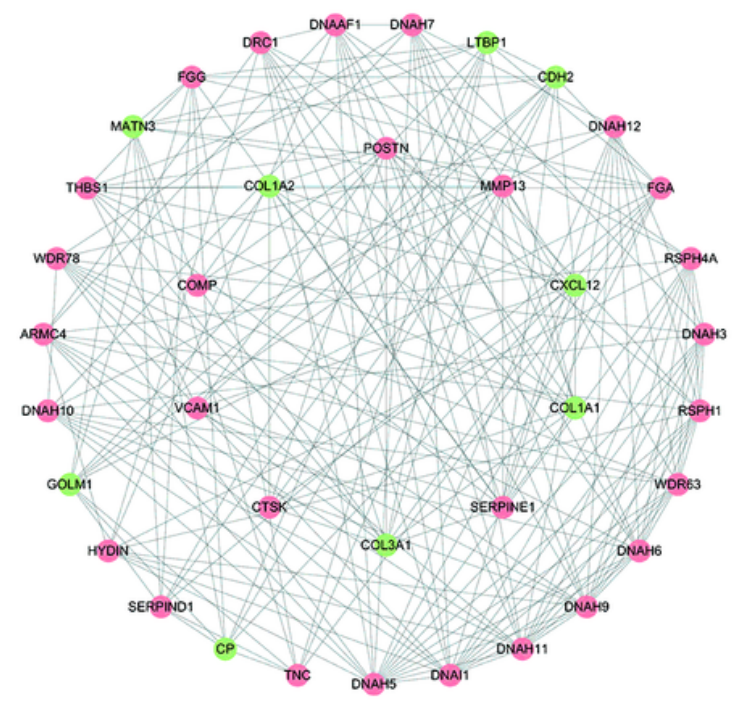

Figure 2

The protein-protein network visualization of the differentially expressed genes. (A) The complete proteinprotein network visualization. (B) The top 2 protein-protein network visualization based on the MCODE 
A

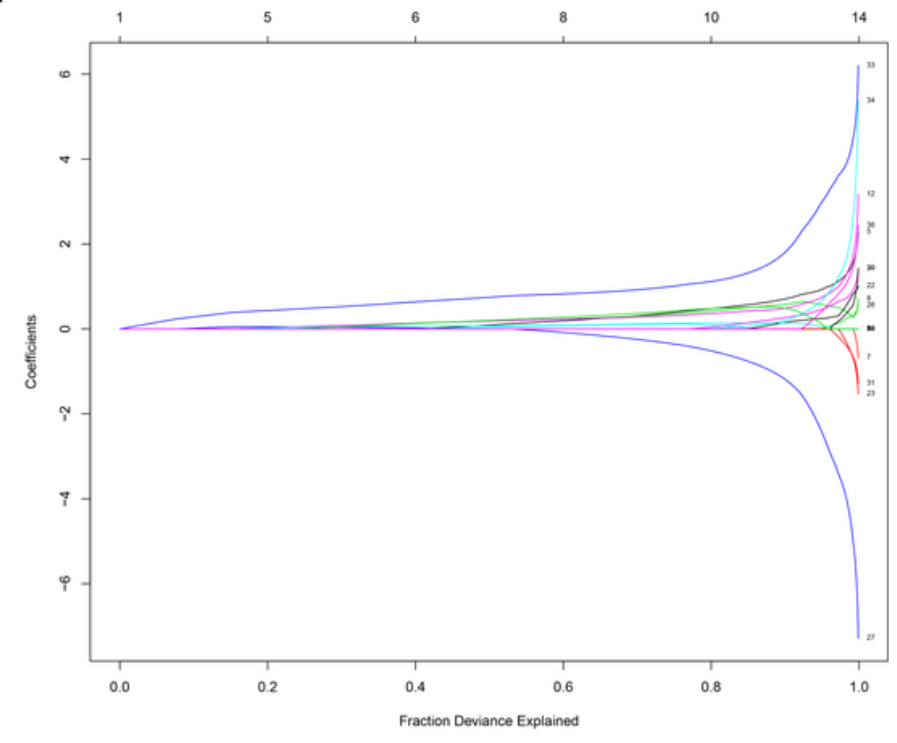

C

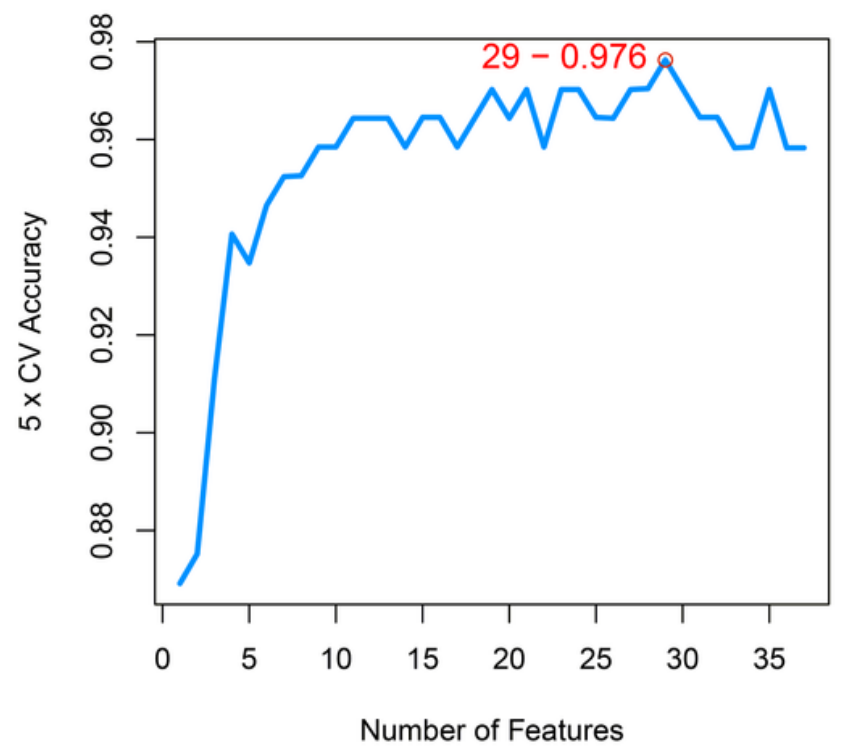

B

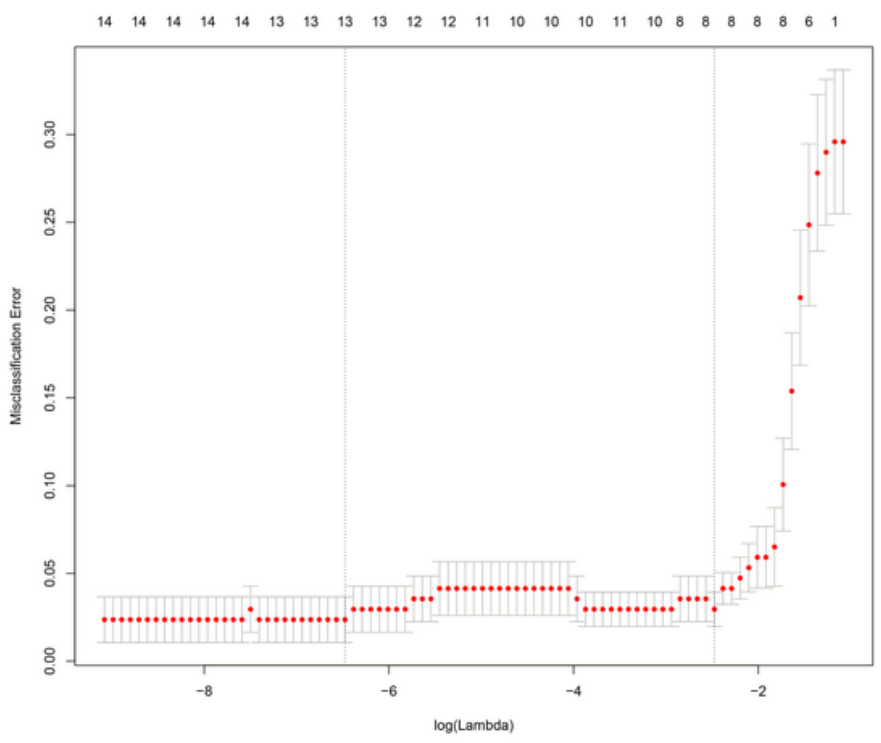

D

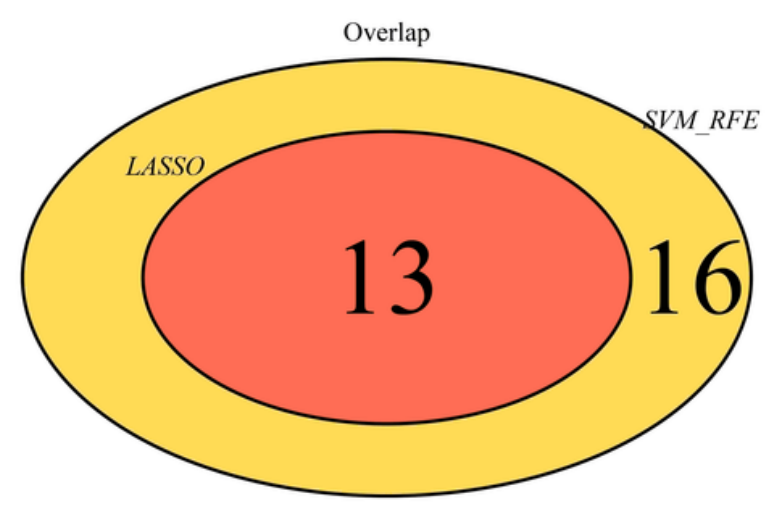

\section{Figure 3}

Selection of the feature genes from two hub modules (A) Least absolute shrinkage and selection operator (LASSO) coefficient profiles of the fractions of genes. (B) 10-fold cross-validation for tuning parameter selection in the LASSO model. (C) Feature gene selection according to recursive feature elimination (RFE). The prediction accuracy versus the number of selected feature genes is plotted as red line. The blue point labels represent the best prediction accuracy (97.6\%), with the corresponding number of selected feature genes being 56. (D) Venn plot for the LASSO model result and SVM-RFE result. 

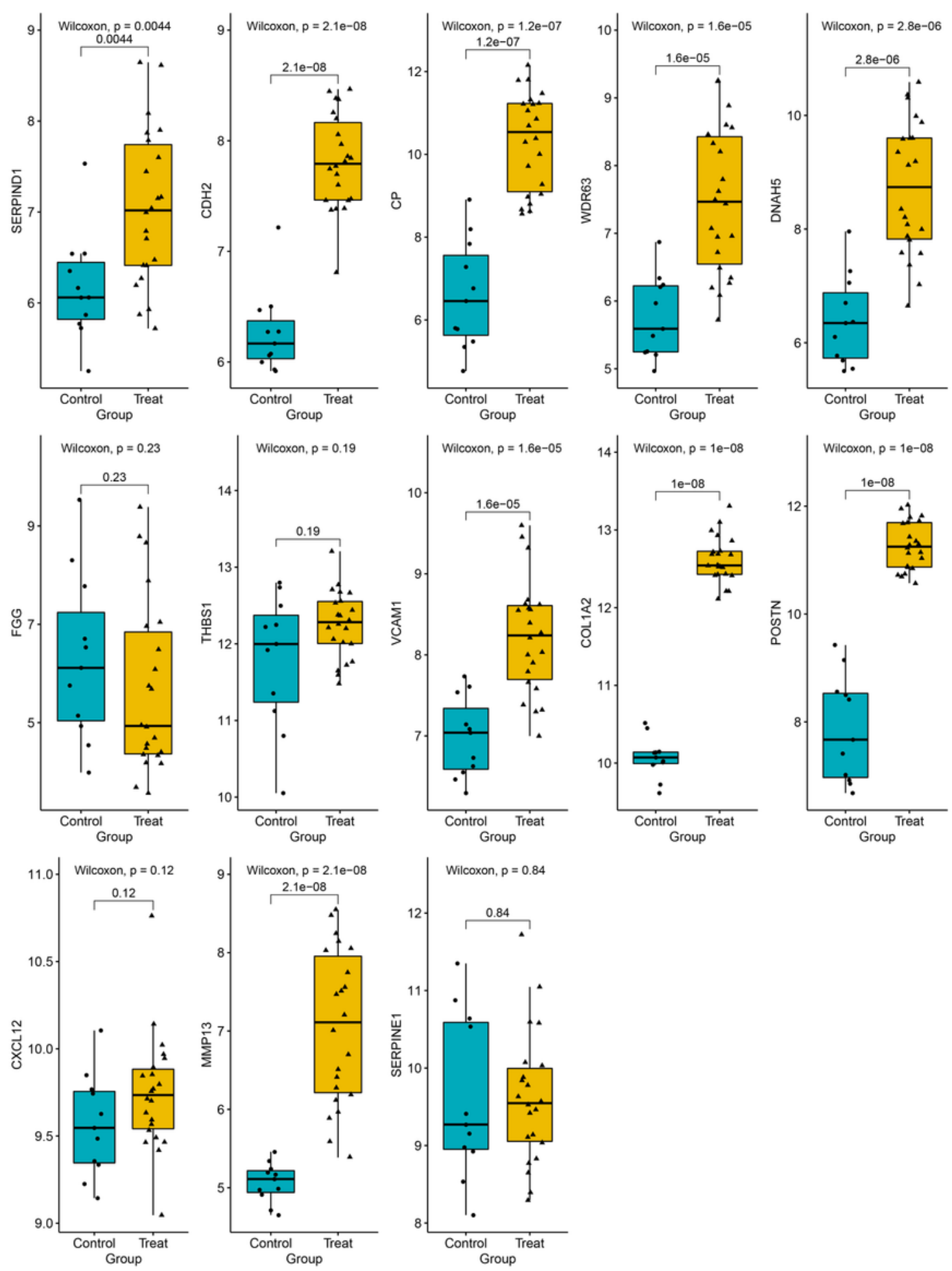

Figure 4

Validation of the 13 key genes expression in the GSE110147 dataset. 

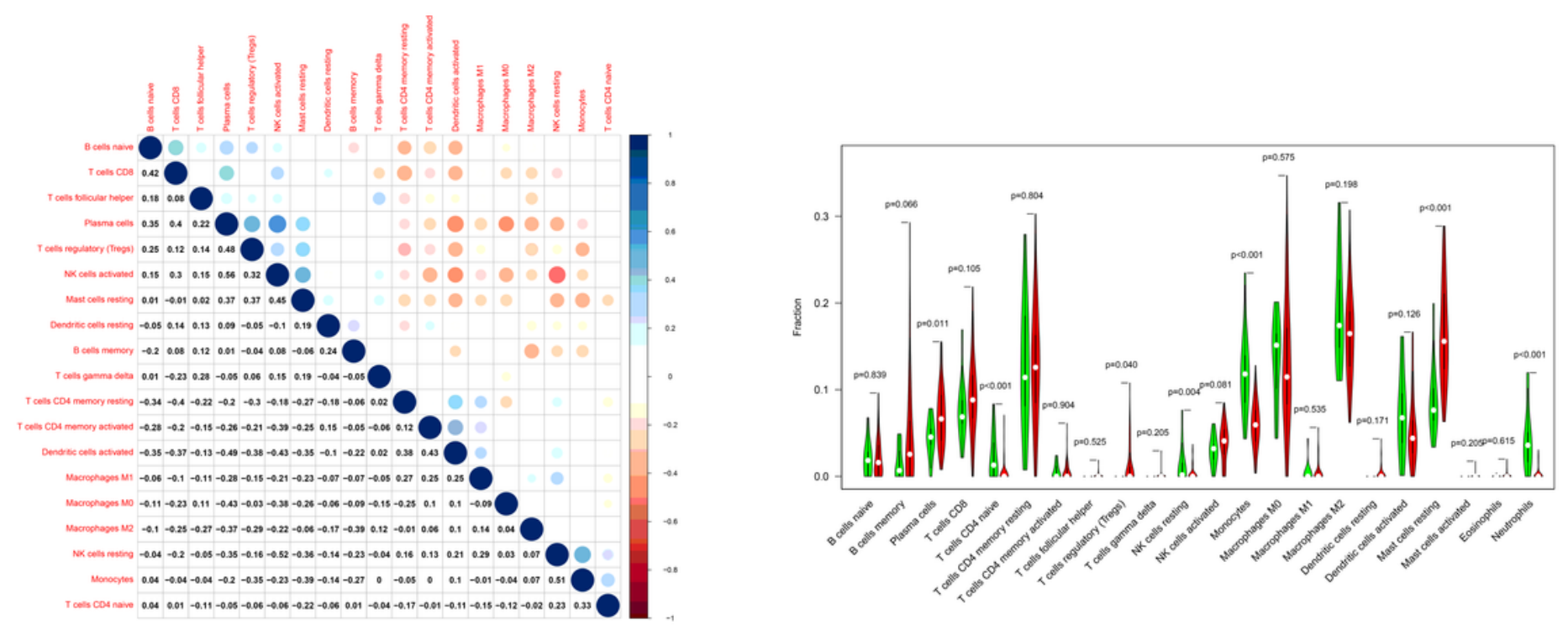

Figure 5

The landscape of the immune cell infiltration level in the IPF tissue. (A) correlation analysis between 22 immune cells. (B) The immune cell infiltration level between normal and IPF tissue. 


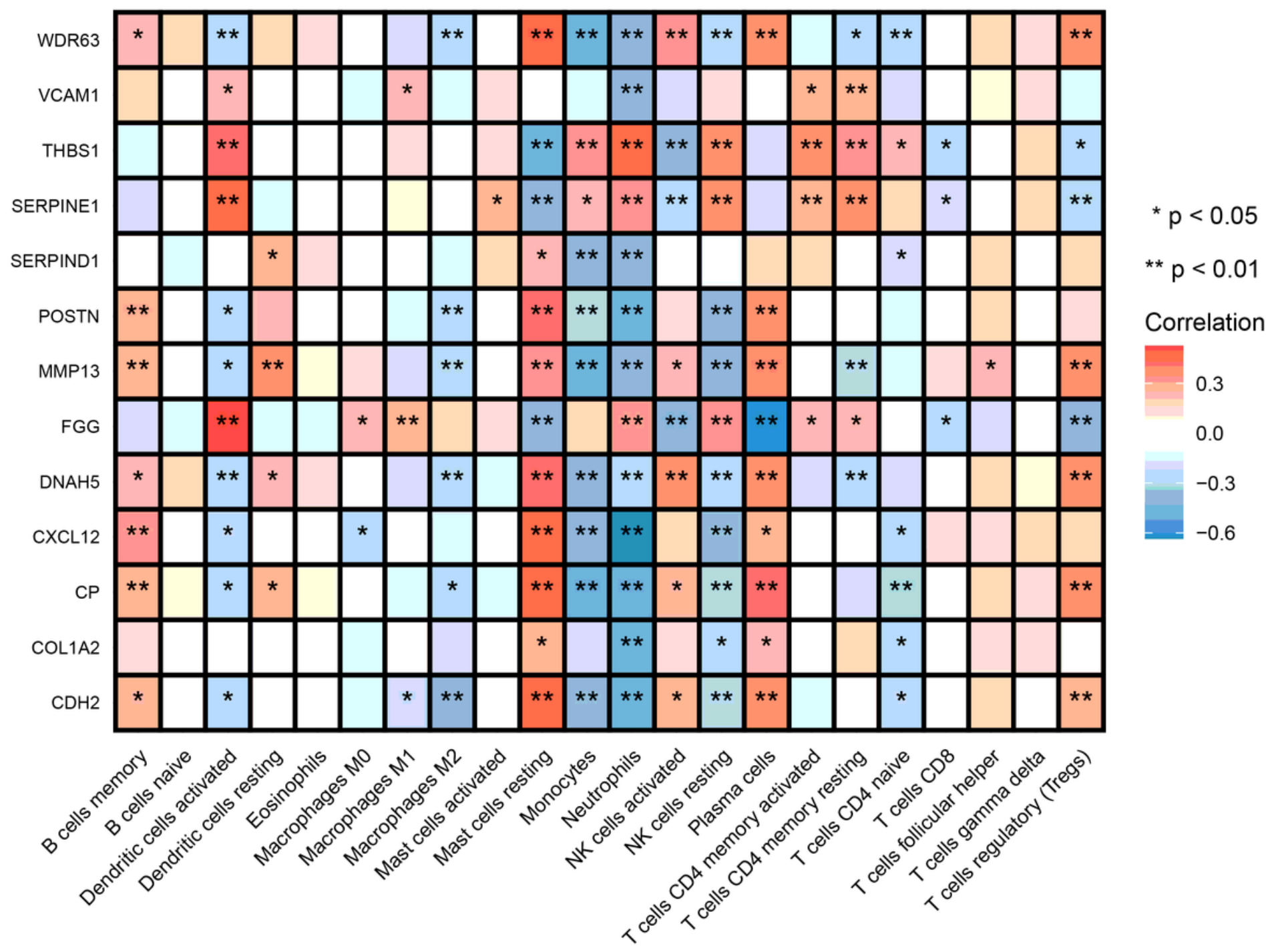

Figure 6

The correlation between 22 immune cells infiltration level and 13 key genes. 


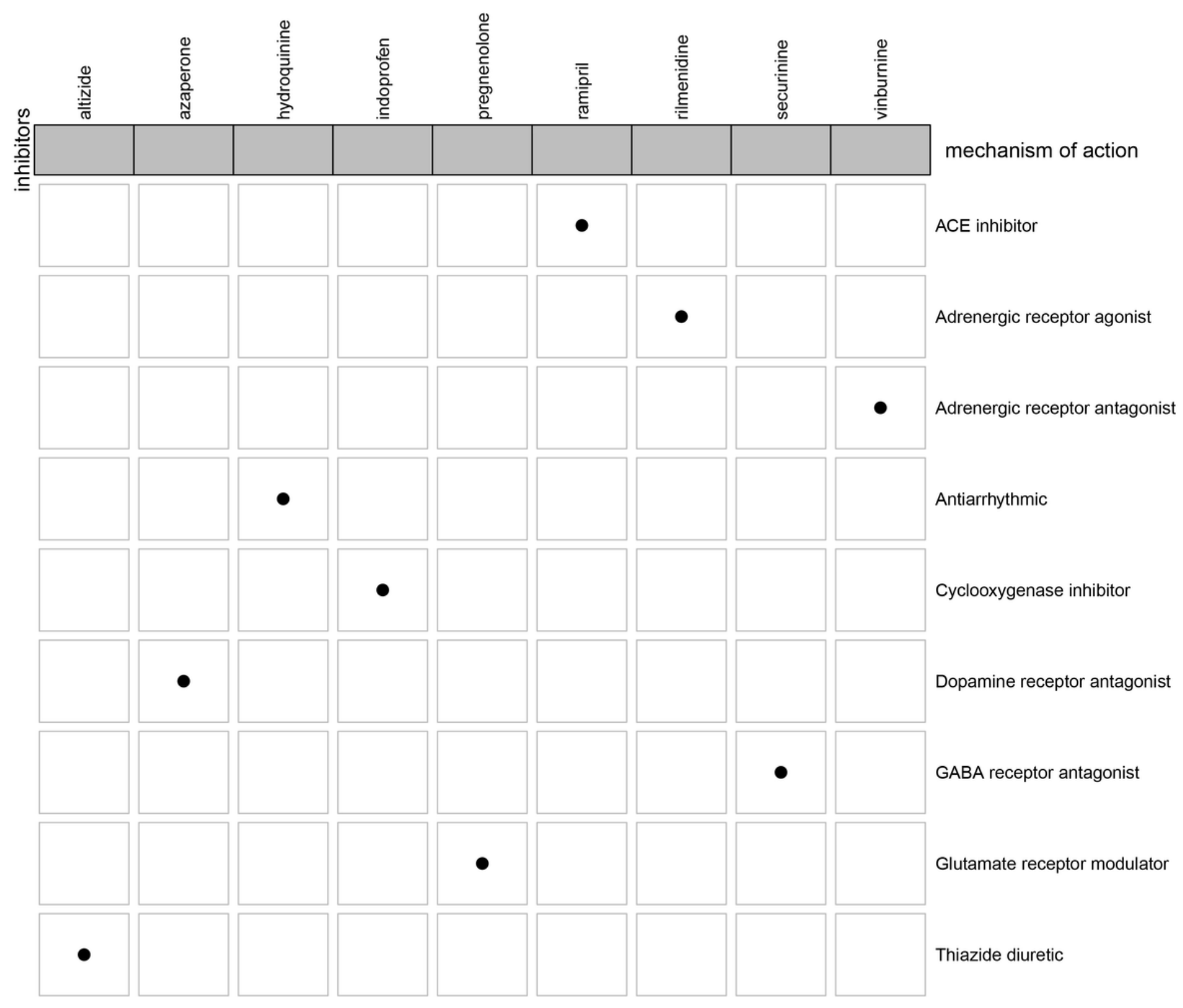

Figure 7

Mechanism of action of potential drugs to the treatment of IPF 